\title{
AVALIAÇÃO DE GENÓTIPOS DE TRITICALE E TRIGO EM AMBIENTES FAVORÁVEIS E DESFAVORÁVEIS NO ESTADO DE SÃO PAULO ${ }^{(1)}$
}

\author{
JOÃO CARLOS FELICIO ${ }^{(2)}$; CARLOS EDUARDO DE OLIVEIRA \\ CAMARGO $^{(2,4)}$; ANTÔNIO WILSON PENTEADO FERREIRA FILHO ${ }^{(2)}$; \\ PAULO BOLLER GALLO ${ }^{(3)}$ \\ RESUMO
}

\begin{abstract}
Avaliou-se a adaptação de 18 genótipos de triticale e dois de trigo em experimentos instalados em dois ambientes, na Estação Experimental de Agronomia do Instituto Agronômico, localizada em Mococa (SP), no período de 1996-99. No primeiro ambiente, aplicou-se a irrigação por aspersão em condição de sequeiro (favorável) e, no segundo, várzea úmida com irrigação por inundação (desfavorável). A diversidade entre os ambientes propiciou diferença entre os padrões de resposta dos genótipos. No ambiente irrigado por aspersão, houve maior produtividade para ambas as espécies, sendo o genótipo de triticale TATU 4/ARDI 1 o que apresentou o melhor rendimento de grãos. Na várzea úmida, destacou-se o triticale LT 978.82/ASAD//TARASCA. O IAC 60 (trigo) apresentou grande variação no rendimento de grãos de um ano para outro, nas condições de várzea. O ciclo das plantas, da emergência ao espigamento, foi mais longo entre os genótipos de triticale nas condições de várzea úmida, quando comparado ao cultivo irrigado por aspersão. Esses resultados indicam que os genótipos de trigo apresentam adaptação específica para o ambiente de sequeiro com irrigação por aspersão, e o grupo de genótipos de triticale, adaptação mais ampla.

Palavras-chave: triticale, rendimento de grãos, adaptabilidade, estabilidade, ciclo das plantas.
\end{abstract}

\section{ABSTRACT \\ EVALUATION OF TRITICALE AND WHEAT GENOTYPES UNDER FAVORABLE AND UNFAVORABLE ENVIRONMENTS IN THE STATE OF SÃO PAULO, BRAZIL}

The environment adaptation of eighteen triticale and two wheat genotypes were evaluated in experiments carried out at two different sites of the Mococa Agronomy Experimental Station, São Paulo State, Brazil, in the period 1996-99. The favorable environment was provided with sprinkler irrigation on an upland; the unfavorable condition consisted of a moist flat area with basin irrigation system. The environment diversity induced different genotypes response patterns. Under the sprinkler irrigation system the yields were higher for both species, but the triticale genotype TATU 4/ARDI 1 presented the highest grain yield. Under moist and flat area condition the triticale LT 978.82/ASAD//TARASCA showed the highest yield. The wheat genotype IAC 60 showed large variations in grain yield in the moist flat area over the two cropping years. The plant cycle from emergence to heading showed higher variation among the triticale genotypes under moist low and flat area in comparison with the upland sprinkler irrigation condition. The results suggested the wheat genotypes presented a specific adaptation for upland with sprinkler irrigation environment and the triticale group of genotypes presented a broader adaptation.

Key words: triticale, grain yield, adaptability, stability, plant cycle.

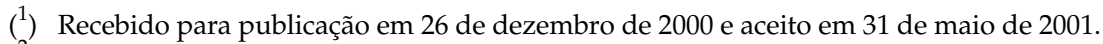

$\left({ }^{2}\right)$ Centro de Plantas Graníferas, Instituto Agronômico (IAC), Caixa Postal 28, 13001-970 Campinas (SP). E-mail: jfelicio@cec.iac.br

(3) Estação Experimental de Agronomia de Mococa, IAC, Caixa Postal 58, 13730-970 Mococa (SP).

(4) Com bolsa de produtividade em pesquisa do CNPq. 


\section{INTRODUÇÃO}

O triticale é, primordialmente, uma fonte energética em rações para o consumo animal. Na Rússia e na Polônia, é semeado em áreas marginais, anteriormente utilizadas para o plantio de centeio; na Austrália, é cultivado para forragem ou pastoreio, em regiões semi-áridas; na Argentina, somente para pastoreio. Na França, Alemanha, Inglaterra e nos Estados Unidos, o triticale é semeado em solos mais pobres, sendo empregado para alimentação animal na forma de feno, silagem da planta ou do grão ou, ainda, como grão seco (BAIER, 1995).

Historicamente, o preço do milho é maior na entressafra, período que coincide com a época da colheita das culturas de inverno e a oferta de grãos de triticale completa o déficit da oferta do milho. Outro aspecto positivo que se deve levar em conta, quando se utiliza o triticale na formulação das rações para aves e suínos, é a mudança do enfoque "déficit de milho" para o de alimentos energéticos (AlbERTONI e BAUERMANN, 1995). De acordo com BAIER (1996), o triticale apresenta 3-4\% mais proteína que o milho, o que permite reduzir o suplemento protéico; no entanto, tem $2-3 \%$ menos gordura, o que lhe confere menor valor energético.

Os genótipos de triticale hoje disponíveis adaptam-se melhor em solos com acidez moderada $(\mathrm{pH}$ entre 4,5 e 5,5 - mais de 3,5\% de matéria orgânica e 5 a $30 \mu \mathrm{mol} \mathrm{de} \mathrm{Al.dm}{ }^{-3}$ de solo) em regiões de altitude superior a $400 \mathrm{~m}$ (temperaturas médias durante o perfilhamento entre $12 \mathrm{e} 14^{\circ} \mathrm{C}$ ).

No Sul do Brasil, os rendimentos médios dos experimentos evoluíram de $2.300 \mathrm{~kg} \cdot \mathrm{ha}^{-1}$ em 1976 para mais de 8.000 kg.ha ${ }^{-1}$ em 1989 (BAIER, 1991). Estudos realizados por Felicio et al. (1988), em condições de várzea na região do Vale do Paraíba, no Estado de São Paulo, mostraram que os genótipos de triticale apresentaram, na média dos anos, produção de grãos superior à dos genótipos de trigo e, também, menor índice de chochamento das espigas.

Segundo Cruz e RegazZi (1994), a manifestação fenotípica é o resultado da interação do genótipo sob a influência do meio em um determinado ambiente. Entretanto, quando se considera uma série de ambientes, os efeitos genéticos e ambientais são proporcionados pela sua interação. As causas também são atribuídas a fatores fisiológicos e bioquímicos próprios de cada genótipo. Como se desenvolvem em sistemas dinâmicos, em que ocorrem constantes mudanças, desde a semeadura até a maturação, há geralmente um comportamento diferenciado dos genótipos em resposta às variações ambientais.
Nesse contexto, o objetivo deste trabalho foi estudar a adaptação de genótipos de triticale em dois ambientes. Considerou-se como favorável o ambiente de sequeiro com irrigação por aspersão e, como desfavorável, o de várzea úmida, com solos de baixa fertilidade natural, drenagem deficiente e altas temperaturas na época do florescimento (FeLICIO et al., 1995).

\section{MATERIAL E MÉTODOS}

Para estudar o efeito do ambiente no rendimento de grãos de triticale, foram instalados experimentos no período de 1996-99, na Estação Experimental de Agronomia de Mococa, do Instituto Agronômico (IAC), seguindo-se as Recomendações da Comissão Técnica de Trigo (CAMPINAS, 1999). Os experimentos foram realizados em dois ambientes, um em condição de irrigação por aspersão (favorável); outro, em várzea irrigada por inundação (desfavorável), com dezoito genótipos de triticale e dois de trigo, no delineamento experimental de blocos ao acaso, com quatro repetições.

Efetuaram-se as semeaduras sempre no terceiro decêndio do mês de abril. Em cada experimento, as parcelas foram compostas por seis linhas de $3 \mathrm{~m}$ de comprimento, com espaçamento de $0,20 \mathrm{~m}$ entre linhas e separação lateral de $0,60 \mathrm{~m}$ entre as parcelas. Procedeu-se à semeadura com 60 sementes viáveis por metro linear de sulco, realizando-se a colheita na área total das parcelas, ou seja, 3,6 $\mathrm{m}^{2}$.

No desenvolvimento das parcelas, procurou-se estabelecer o máximo de uniformidade, mantendo-se as plantas livres de competição com ervas daninhas.

A adubação mineral foi feita a lanço, com posterior incorporação ao solo, antes da semeadura. A quantidade de fertilizante foi aplicada de acordo com as recomendações de RAIJ et al. (1996) e com os resultados das análises químicas do solo. Para adubação em cobertura, aplicaram-se 40 kg.ha- ${ }^{-1}$ de N após 20-30 dias da emergência das plântulas.

Para a irrigação por aspersão, adotou-se o método proposto por Silva et al. (1984); na zona tritícola H, onde se realizou a pesquisa, o cultivo de trigo apenas será viável se houver irrigação (CAMPINAS, 1999). Aplicaram-se 40-60 mm de água, logo após a semeadura, a fim de umedecer o perfil do solo, quando se instalaram os tensiômetros em diferentes pontos, à profundidade de $12 \mathrm{~cm}$. As irrigações complementares foram realizadas quando a média das leituras dos tensiômetros indicava $-0,06 \mathrm{MPa}$; determinou-se a lâmina líquida aplicada pela evaporação acumulada, medida no tanque classe $\mathrm{A}$, entre os intervalos de irrigação.

Na várzea, procedeu-se à irrigação pelo sistema de inundação ou banhos rápidos, conforme utilizado 
para a cultura do arroz (altura da lâmina de água de 3 a $8 \mathrm{~cm}$ ), que consiste em cobrir com água toda a área do tabuleiro, fazendo-se escorrer o excesso, esgotando-a no ponto de maior desnível (SiLva et al., 1980).

Efetuou-se a análise, considerando-se separada e conjuntamente, os ensaios com irrigação por aspersão e os semeados na várzea para avaliar o efeito do ano, do ambiente, dos genótipos e de suas interações (GOMEs, 1970). Estudaram-se a estabilidade e a adaptabilidade pelo método proposto por EBERHART e Russell (1966), com base nos coeficientes de cada genótipo em relação ao índice ambiental, para cada ano, no período de 1996-99, considerando como genótipo ideal aquele que apresentasse alto rendimento médio de grãos, coeficiente de regressão igual a 1,0 $(b=1)$ e desvio de regressão igual a zero $\left(S^{2} d=0\right)$.

O subperíodo emergência-espigamento foi determinado pelo número de dias decorridos da emergência das plântulas ao espigamento (florescimento). Os genótipos com ciclo da emergência ao espigamento entre 56-60, 61-65 e acima de 65 dias foram considerados, respectivamente, como precoce, médio e tardio.

A altura das plantas foi medida no campo, na época de maturação, tomando-se a distância, em centímetros, do nível do solo ao ápice da espiga, com exclusão das aristas, e estimando-se a média de diferentes pontos em cada parcela.

Ao acamamento atribuíram-se notas de 0 (nenhuma planta acamada) a 100 (todas as plantas acamadas) em cada parcela, por avaliação visual realizada na época da maturação.

\section{RESULTADOS E DISCUSSÃO}

A germinação das sementes e o estande inicial dos experimentos, nos anos e ambientes considerados, não apresentaram falhas que resultassem em prejuízo no rendimento final de grãos, mesmo considerando que a germinação em solos de várzea é, geralmente, prejudicada pelo excesso de umidade.

Os quadrados médios das análises da variância para rendimento de grãos dos genótipos de triticale e de trigo, obtidos nos dois ambientes (sequeiro irrigado por aspersão e várzea irrigada por inundação), e as interações ano $x$ ambiente, ano $x$ genótipo, ambiente $x$ genótipo e a interação tripla ano $x$ ambiente $x$ genótipo referente aos experimentos instalados em Mococa (SP), no período de 1996-99, encontram-se no quadro 1.

No quadro 2 são apresentados os quadrados médios da análise da variância para rendimento de

Quadro 1. Análise da variância, em conjunto e separadamente para os dois ambientes, para rendimento de grãos dos genótipos de triticale e de trigo, avaliados em Mococa (SP), em área de sequeiro com irrigação e em várzea úmida, em 1996-99

\begin{tabular}{lccccc}
\hline Causas da variação & GL & $\begin{array}{c}\text { QM } \\
\text { Irrigado e várzea }\end{array}$ & GL & $\begin{array}{c}\text { QM } \\
\text { Irrigado }\end{array}$ & $\begin{array}{c}\text { QM } \\
\text { Várzea }\end{array}$ \\
\hline Repetição & 3 & - & 3 & - & - \\
Ano (A) & 3 & $36.778 .254,44^{* *}$ & 3 & $18.181 .306,09^{* *}$ & $33.679 .612,86^{* *}$ \\
Resíduo A & 9 & $385.327,17$ & 9 & $807.629,28$ & $405.163,20$ \\
Ambiente (B) & 1 & $374.815 .695,64^{* *}$ & - & - & - \\
Genótipo (G) & 19 & $3.178 .625,55^{* *}$ & 19 & $1.595 .524,63^{* *}$ & $4.083 .605,19^{* *}$ \\
A x B & 3 & $15.082 .664,50^{* *}$ & - & - & - \\
A x G & 57 & $1.038 .825,50^{* *}$ & 57 & $620.369,23^{* *}$ & $1.521 .114,03^{* *}$ \\
B x G & 19 & $2.500 .504,26^{* *}$ & - & - & - \\
A x B x G & 57 & $1.102 .657,76^{* *}$ & - & - & - \\
Resíduo B & 468 & $409.077,23$ & 228 & $349.471,36$ & $452.413,94$ \\
\hline
\end{tabular}

**: Significativo ao nível de $1 \%$ pelo teste F.

Quadro 2. Quadrado médio da análise da variância para cada ano considerando os experimentos realizados nos dois ambientes para rendimento de grãos dos genótipos de triticale e de trigo avaliados em Mococa (SP), em 1996-99

\begin{tabular}{lrcccc}
\hline \multirow{2}{*}{ Causas da variação } & \multirow{2}{*}{ GL } & \multicolumn{4}{c}{ Anos } \\
\cline { 3 - 6 } & 1 & $150.335 .131,92^{* *}$ & $28.317 .318,11^{* *}$ & $184.133 .348,63^{* *}$ & $57.277 .890,50^{* *}$ \\
Ambiente (A) & 1 & $496.105,01$ & $567.468,59$ & $97.363,91$ & $711.827,81$ \\
Resíduo A & 19 & $994.887,10 \mathrm{~ns}$ & $2.615 .039,52^{* *}$ & $824.224,60^{* *}$ & $1.860 .950,84^{* *}$ \\
Genótipo (G) & 19 & $740.076,11 \mathrm{~ns}$ & $2.678 .251,13^{* *}$ & $625.458,18^{* *}$ & $1.764 .692,17^{* *}$ \\
A x G & 114 & $668.262,87$ & $415.287,13$ & $157.869,26$ & $362.351,35$ \\
Resíduo B & & &
\end{tabular}

**: Significativo ao nível de $1 \%$ pelo teste F. ns: Não significativo. 
grãos, considerando-se cada ano, separadamente, nos dois ambientes a fim de verificar o efeito de ambiente, genótipo e interação genótipo $x$ ambiente.

Nas análises da variância, verificou-se o efeito significativo para todas as causas de variação ao nível de $1 \%$. Os genótipos estudados apresentaram variações significativas para rendimento de grãos, de acordo com o ano e o ambiente em que foram avaliados. Apesar do efeito significativo entre ambientes e para as interações genótipo $\mathrm{x}$ ambiente e genótipo $\mathrm{x}$ ano, os resultados são semelhantes aos observados por
BAKER (1969), pois a adaptabilidade de um genótipo pode sobrepor-se a essas variações.

Alguns genótipos apresentaram alto rendimento nos dois ambientes, como triticale LT 978.82/ ASAD//TARASCA que mostrou média superior de rendimento de grãos na várzea e no ambiente com irrigação por aspersão (Quadros 3 e 4).

No ambiente irrigado por aspersão (Quadro 3), destacou-se o triticale TATU 4/ARDI 1 com o melhor rendimento de grãos, não diferindo, porém, dos genótipos LT 978.82/ASAD/ /TARASCA, BAGAL 7,

Quadro 3. Rendimento médio em kg.ha ${ }^{-1}$, avaliação da estabilidade e adaptabilidade de acordo com o modelo de EBERHART e RUSSEL (1966) e coeficiente de determinação $\left(R^{2}\right)$ para os genótipos de triticale avaliados em condição de irrigação por aspersão, em Mococa (Zona H), Estado de São Paulo, de 1996 a 1999

\begin{tabular}{|c|c|c|c|c|c|c|c|c|}
\hline \multirow{2}{*}{ Genótipos } & \multicolumn{4}{|c|}{ Anos } & \multirow{2}{*}{ Média $^{(1)}$} & \multirow{2}{*}{$\beta_{1}^{(2)}$} & \multirow{2}{*}{$S^{2} d^{(3)}$} & \multirow{2}{*}{$\mathrm{R}^{2}$} \\
\hline & 1996 & 1997 & 1998 & 1999 & & & & \\
\hline & 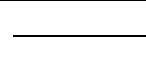 & +2 & - kg.ha ${ }^{-1}$ & 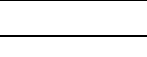 & $\overline{ }$ & & kg.ha ${ }^{-1}$ & $\%$ \\
\hline IAC 3 (Banteng) & $5.124 \mathrm{ab}$ & $3.434 c-e$ & 3.708 a-d & $3.829 \mathrm{~cd}$ & $4.024 \mathrm{~b}-\mathrm{d}$ & 1,50 & - & 90,76 \\
\hline STIER “S” & $4.764 \mathrm{a}-\mathrm{c}$ & $3.469 \mathrm{c}-\mathrm{e}$ & $3.497 \mathrm{~b}-\mathrm{d}$ & $3.947 \mathrm{~cd}$ & $3.919 \mathrm{~cd}$ & 1,26 & - & 99,48 \\
\hline IAC 2 (Tarasca) & $5.242 \mathrm{ab}$ & 3.981 a-e & $3.708 \mathrm{a}-\mathrm{d}$ & $4.314 \mathrm{a}-\mathrm{c}$ & $4.311 \mathrm{a}-\mathrm{c}$ & 1,37 & - & 95,86 \\
\hline ARDILLA & $4.965 \mathrm{ab}$ & $3.926 \mathrm{~b}-\mathrm{e}$ & 3.798 a-d & $4.999 \mathrm{ab}$ & $4.422 \mathrm{a}-\mathrm{c}$ & 1,16 & $284 \mathrm{~ns}$ & 73,37 \\
\hline IAC 1 & $4.785 \mathrm{a}-\mathrm{c}$ & 3.801 b-e & $3.923 \mathrm{a}-\mathrm{c}$ & $3.857 \mathrm{~cd}$ & $4.091 \mathrm{~b}-\mathrm{d}$ & 0,87 & - & 80,59 \\
\hline ICT 8803 & $4.854 \mathrm{a}-\mathrm{c}$ & $3.483 \mathrm{c}-\mathrm{e}$ & $3.600 \mathrm{a}-\mathrm{d}$ & $4.369 \mathrm{a}-\mathrm{c}$ & $4.077 \mathrm{~b}-\mathrm{d}$ & 1,33 & - & 95,56 \\
\hline IAPAR 23 & $3.940 \mathrm{bc}$ & $3.393 \mathrm{c}-\mathrm{e}$ & $3.725 \mathrm{a}-\mathrm{d}$ & $3.871 \mathrm{~cd}$ & $3.732 \mathrm{~d}$ & 0,39 & - & 60,79 \\
\hline CEP 15 & $3.510 \mathrm{c}$ & $2.956 \mathrm{e}$ & $3.199 \mathrm{~d}$ & $3.296 \mathrm{~d}$ & $3.240 \mathrm{e}$ & 0,43 & - & 81,77 \\
\hline $\begin{array}{l}\text { CML"S" /PATO//KISS/DNF/ } \\
\text { 3/BGL"S" / 4/ANTEATER }\end{array}$ & $4.992 \mathrm{ab}$ & $4.175 \mathrm{a}-\mathrm{c}$ & 3.940 a-c & $4.438 \mathrm{a}-\mathrm{c}$ & $4.386 \mathrm{a}-\mathrm{c}$ & 0,92 & - & 94,94 \\
\hline IAC $289(t)$ & $4.335 \mathrm{a}-\mathrm{c}$ & $4.986 \mathrm{a}$ & $3.898 \mathrm{a}-\mathrm{c}$ & $4.099 \mathrm{a}-\mathrm{d}$ & $4.329 \mathrm{a}-\mathrm{c}$ & $0,14^{* *}$ & $490^{*}$ & 2,26 \\
\hline LT 978.82/ASAD/ /TARASCA & $5.512 \mathrm{a}$ & $4.681 \mathrm{ab}$ & 3.729 a-d & $3.836 \mathrm{~cd}$ & $4.439 \mathrm{a}-\mathrm{c}$ & 1,18 & $674^{* *}$ & 46,46 \\
\hline TATU 4/ARDI 1 & $5.152 \mathrm{ab}$ & $4.099 \mathrm{a}-\mathrm{d}$ & $4.168 \mathrm{ab}$ & $5.076 \mathrm{a}$ & $4.624 \mathrm{a}$ & 1,06 & $89 \mathrm{~ns}$ & 80,30 \\
\hline TARASCA 87/YOGUI "S" & $5.283 \mathrm{ab}$ & $3.670 \mathrm{~b}-\mathrm{e}$ & 3.659 a-d & $4.522 \mathrm{a}-\mathrm{c}$ & $4.283 \mathrm{a}-\mathrm{c}$ & 1,62 & - & 98,90 \\
\hline $\begin{array}{l}\text { BGL/CIN//MUS/4/DLF99/ } \\
3 / \mathrm{M}^{2} \mathrm{~A} / \mathrm{F} 3\end{array}$ & $4.778 \mathrm{a}-\mathrm{c}$ & 3.794 b-e & $4.227 \mathrm{a}$ & $4.556 \mathrm{a}-\mathrm{c}$ & $4.339 \mathrm{a}-\mathrm{c}$ & 0,79 & - & 77,51 \\
\hline BAGAL 7 & $5.228 \mathrm{ab}$ & $4.092 \mathrm{a}-\mathrm{d}$ & $4.241 \mathrm{a}$ & $4.501 \mathrm{a}-\mathrm{c}$ & $4.515 \mathrm{ab}$ & 1,04 & - & 97,36 \\
\hline $\begin{array}{l}\text { CML/PATO//BGL/3/ITA } \\
\text { BULK/4/DRIRA/FAS204/ } \\
\text { 5/YOGUI "S" }\end{array}$ & $4.702 \mathrm{a}-\mathrm{c}$ & $3.067 \mathrm{de}$ & $3.701 \mathrm{a}-\mathrm{d}$ & $4.286 \mathrm{a}-\mathrm{d}$ & $3.939 \mathrm{~cd}$ & 1,35 & $221 \mathrm{~ns}$ & 82,05 \\
\hline MUS“S”*2/HARE 57 & $4.764 \mathrm{a}-\mathrm{c}$ & $3.289 \mathrm{c}-\mathrm{e}$ & $3.864 \mathrm{a}-\mathrm{d}$ & $4.182 \mathrm{a}-\mathrm{d}$ & $4.025 \mathrm{~b}-\mathrm{d}$ & 1,19 & - & 85,71 \\
\hline GRADO/ARDI 1//CIVET & $4.660 \mathrm{a}-\mathrm{c}$ & $2.915 \mathrm{e}$ & 3.923 a-c & $4.362 \mathrm{a}-\mathrm{c}$ & $3.965 c-d$ & 1,28 & $469^{*}$ & 64,77 \\
\hline HARE 263/CIVET"S" & $4.951 \mathrm{ab}$ & 3.171 c-e & $3.684 \mathrm{a}-\mathrm{d}$ & $4.002 \mathrm{~b}-\mathrm{d}$ & $3.952 \mathrm{~cd}$ & 1,51 & - & 92,51 \\
\hline IAC $60(t)$ & $4.113 \mathrm{a}-\mathrm{c}$ & $5.013 \mathrm{a}$ & $3.479 \mathrm{~cd}$ & $3.843 \mathrm{~cd}$ & $4.112 \mathrm{a}-\mathrm{c}$ & $0,18^{* *}$ & $737^{* *}$ & 1,81 \\
\hline Média & $4.783 \mathrm{~A}$ & $3.770 \mathrm{C}$ & $3.783 \mathrm{C}$ & $4.209 \mathrm{~B}$ & 4.136 & & & \\
\hline
\end{tabular}

${ }^{1}$ ) Médias da produtividade de grãos de genótipos em cada ano e na média geral, em letras minúsculas; médias para comparação entre anos, em letras maiúsculas. Médias seguidas por letras distintas diferem entre si pelo teste de Duncan ao nível de 5\%.

$\left({ }^{2}\right)$ Coeficiente de regressão linear; ${ }^{*} \mathrm{e}^{* *}$ : significativo a $5 \%$ e a $1 \%$ de probabilidade, respectivamente, pelo teste de Tukey.

( ${ }^{3}$ ) Desvio de regressão; ${ }^{*} \mathrm{e}^{* *}$ : significativo a $5 \%$ e a $1 \%$ de probabilidade, respectivamente, pelo teste $\mathrm{F}$.

(t) Trigo. -: Desvio de regressão menor que o erro experimental. ns: Não significativo. 
Quadro 4. Rendimento médio em kg.ha ${ }^{-1}$, avaliação da estabilidade e adaptabilidade de acordo com o modelo de EBERHART e RUSSEL (1966) e coeficiente de determinação $\left(R^{2}\right)$ para os genótipos de triticale avaliados em condição de várzea úmida, em Mococa (Zona H), Estado de São Paulo, de 1996 a 1999

\begin{tabular}{|c|c|c|c|c|c|c|c|c|}
\hline \multirow{2}{*}{ Genótipos } & \multicolumn{4}{|c|}{ Anos } & \multirow{2}{*}{ Média $^{(1)}$} & \multirow{2}{*}{$\beta_{1}^{(2)}$} & \multirow{2}{*}{$\mathrm{S}^{2} \mathrm{~d}^{(3)}$} & \multirow{2}{*}{$\mathrm{R}^{2}$} \\
\hline & 1996 & 1997 & 1998 & 1999 & & & & \\
\hline & \multicolumn{5}{|c|}{$\longrightarrow$ kg.ha ${ }^{-1}$} & \multicolumn{3}{|c|}{ kg.ha ${ }^{-1}$} \\
\hline IAC 3 (Banteng) & $3.303 \mathrm{a}-\mathrm{c}$ & $3.220 \mathrm{bc}$ & $2.326 \mathrm{ab}$ & $3.718 \mathrm{ab}$ & $3.142 \mathrm{a} b$ & 0,86 & - & 93,93 \\
\hline STIER “S” & $2.790 \mathrm{a}-\mathrm{c}$ & $2.008 \mathrm{de}$ & $1.163 \mathrm{ef}$ & $2.139 \mathrm{e}-\mathrm{g}$ & $2.025 \mathrm{e}$ & 0,83 & 339 ns & 65,99 \\
\hline IAC 2 (Tarasca) & $2.714 \mathrm{a}-\mathrm{c}$ & $3.677 \mathrm{~b}$ & $1.938 \mathrm{a}-\mathrm{d}$ & $3.510 \mathrm{a}-\mathrm{c}$ & $2.960 \mathrm{bc}$ & 1,09 & $296 \mathrm{~ns}$ & 79,10 \\
\hline ARDILLA & $2.860 \mathrm{a}-\mathrm{c}$ & $3.310 \mathrm{bc}$ & $2.333 \mathrm{ab}$ & $2.894 \mathrm{~b}-\mathrm{f}$ & $2.849 \mathrm{bc}$ & 0,52 & - & 73,72 \\
\hline IAC 1 & $2.825 \mathrm{a}-\mathrm{c}$ & $3.233 \mathrm{bc}$ & $2.112 \mathrm{a}-\mathrm{c}$ & $4.397 \mathrm{a}$ & $3.142 \mathrm{ab}$ & 1,15 & $644^{*}$ & 61,55 \\
\hline ICT 8803 & $2.333 \mathrm{bc}$ & $3.822 \mathrm{~b}$ & $1.668 \mathrm{c}-\mathrm{e}$ & $3.704 \mathrm{ab}$ & $2.882 \mathrm{bc}$ & 1,33 & $659^{* *}$ & 67,15 \\
\hline IAPAR 23 & $3.240 \mathrm{a}-\mathrm{c}$ & $2.853 \mathrm{~b}-\mathrm{d}$ & 1.807 a-e & $3.420 \mathrm{a}-\mathrm{d}$ & $2.830 \mathrm{bc}$ & 1,05 & - & 90,27 \\
\hline CEP 15 & $2.146 \mathrm{c}$ & $3.836 \mathrm{~b}$ & $1.336 \mathrm{~d}-\mathrm{f}$ & $2.451 \mathrm{~d}-\mathrm{g}$ & 2.442 c-e & 1,15 & $823^{* *}$ & 51,41 \\
\hline $\begin{array}{l}\text { CML“S" / PATO//KISS/DNF/ } \\
\text { 3/BGL"S" / } / \text { ANTEATER }\end{array}$ & $3.580 \mathrm{ab}$ & $3.005 \mathrm{~b}-\mathrm{d}$ & $1.731 \mathrm{~b}-\mathrm{e}$ & $3.185 \mathrm{~b}-\mathrm{d}$ & $2.875 \mathrm{bc}$ & 1,14 & $147 \mathrm{~ns}$ & 85,94 \\
\hline IAC $289(t)$ & $2.042 \mathrm{c}$ & $3.192 \mathrm{bc}$ & 1.662 c-e & $2.077 \mathrm{fg}$ & $2.243 \mathrm{de}$ & 0,59 & $561^{*}$ & 34,40 \\
\hline LT 978.82/ASAD/ /TARASCA & $3.088 \mathrm{a}-\mathrm{c}$ & $4.840 \mathrm{a}$ & $2.402 \mathrm{a}$ & $3.898 \mathrm{ab}$ & $3.557 \mathrm{a}$ & 1,23 & $763^{* *}$ & 58,02 \\
\hline TATU 4/ARDI 1 & $2.333 \mathrm{bc}$ & $3.566 \mathrm{bc}$ & $1.710 \mathrm{~b}-\mathrm{e}$ & $2.555 \mathrm{c}-\mathrm{g}$ & $2.541 \mathrm{~cd}$ & 0,86 & $554^{*}$ & 52,84 \\
\hline TARASCA 87/YOGUI “S” & $3.033 \mathrm{a}-\mathrm{c}$ & $2.077 \mathrm{de}$ & $1.274 \mathrm{~d}-\mathrm{f}$ & $3.898 \mathrm{ab}$ & $2.570 \mathrm{~cd}$ & 1,38 & $781^{* *}$ & 61,82 \\
\hline $\begin{array}{l}\text { BGL/CIN//MUS/4/DLF99/ } \\
\text { 3/M²A/F3 }\end{array}$ & $2.603 \mathrm{a}-\mathrm{c}$ & 2.527 c-e & $1.433 \mathrm{~d}-\mathrm{f}$ & $1.800 \mathrm{~g}$ & $2.091 \mathrm{de}$ & 0,61 & $360 \mathrm{~ns}$ & 49,84 \\
\hline BAGAL 7 & $2.860 \mathrm{a}-\mathrm{c}$ & $2.880 \mathrm{~b}-\mathrm{d}$ & $824 \mathrm{fg}$ & $3.213 \mathrm{~b}-\mathrm{d}$ & $2.444 \mathrm{c}-\mathrm{e}$ & $1,67^{*}$ & - & 99,46 \\
\hline $\begin{array}{l}\text { CML/PATO//BGL/3/ITA } \\
\text { BULK/4/DRIRA/ }\end{array}$ & & & & & & & & \\
\hline FAS204/5/YOGUI “S” & $2.756 \mathrm{a}-\mathrm{c}$ & $1.544 \mathrm{e}$ & $1.378 \mathrm{~d}-\mathrm{f}$ & $3.170 \mathrm{~b}-\mathrm{d}$ & $2.214 \mathrm{de}$ & 0,87 & $759^{* *}$ & 41,45 \\
\hline MUS “S”*2/HARE 57 & $3.150 \mathrm{a}-\mathrm{c}$ & $3.642 \mathrm{~b}$ & $1.890 \mathrm{a}-\mathrm{d}$ & $3.088 \mathrm{~b}-\mathrm{e}$ & $2.943 \mathrm{bc}$ & 1,07 & - & 87,26 \\
\hline GRADO/ARDI 1//CIVET & $2.756 \mathrm{a}-\mathrm{c}$ & $2.846 \mathrm{~b}-\mathrm{d}$ & $2.306 a-c$ & $3.663 \mathrm{ab}$ & $2.892 \mathrm{bc}$ & 0,65 & $302 \mathrm{~ns}$ & 57,35 \\
\hline HARE 263/CIVET “S” & $2.714 \mathrm{a}-\mathrm{c}$ & $2.098 \mathrm{de}$ & 1.170 ef & $2.589 \mathrm{c}-\mathrm{g}$ & 2.143 de & 0,98 & $103 \mathrm{~ns}$ & 83,21 \\
\hline IAC $60(t)$ & $3.753 \mathrm{a}$ & $394 \mathrm{f}$ & $297 \mathrm{~g}$ & $872 \mathrm{~h}$ & $1.329 \mathrm{f}$ & 0,86 & $1.852^{* *}$ & 11,69 \\
\hline Média & $2.844 \mathrm{~B}$ & $2.928 \mathrm{~A}$ & $1.638 \mathrm{C}$ & $3.013 \mathrm{~A}$ & 2.606 & & & \\
\hline
\end{tabular}

$\left({ }^{1}\right)$ Médias da produtividade de grãos de genótipos em cada ano e na média geral em letras minúsculas, e médias para comparação entre anos em letras maiúsculas. Médias seguidas por letras distintas diferem entre si pelo teste de Duncan ao nível de $5 \%$.

$\left({ }^{2}\right)$ Coeficiente de regressão linear; ${ }^{*} \mathrm{e}^{* *}$ : significativo a $5 \%$ e a $1 \%$ de probabilidade, respectivamente, pelo teste de Tukey.

(3) Desvio de regressão; ${ }^{*}{ }^{* *}$ : significativo a $5 \%$ e a $1 \%$ de probabilidade, respectivamente, pelo teste F.

(t) Trigo. -: Desvio de regressão menor que o erro experimental. ns: Não significativo.

IAC 2, TARASCA 87/YOGUI"S", CML"S" /PATO/ /KISS/DNF/3/BGL"S"/4/ANTEATER, ARDILLA e BGL/CIN/ / MUS/4/DLF99/3/M²A/F3 e os trigos IAC 289 e IAC 60.

No ambiente de várzea úmida, o genótipo de triticale LT 978.82/ ASAD/ / TARASCA foi o mais produtivo, mas não diferiu estatisticamente do IAC 3 e IAC 1 (Quadro 4).

A análise da estabilidade e adaptabilidade, realizada com os dados dos quatro anos para o ambiente com irrigação por aspersão, mostrou variabilidade entre os genótipos (Quadro 3). Os trigos IAC 60 e IAC 289 apresentaram desvio de regressão significativos a $1 \%$ e a $5 \%$ respectivamente e b $<1$ (negativo), indicativo da adaptabilidade específica a ambiente desfavorável, além de acentuada variação no rendimento.

O genótipo TATU 4/ARDI 1 apresentou média elevada para rendimento e $b=1(1,06)$, indicando ampla adaptabilidade, resposta à melhoria do ambiente e alto rendimento quando o ambiente foi desfavorável. 
Quadro 5. Número de dias entre a emergência e o espigamento, ciclo e altura das plantas dos genótipos de triticale e trigo avaliados em condição de irrigação por aspersão, em Mococa (Zona H), Estado de São Paulo, de 1996 a 1999

\begin{tabular}{|c|c|c|c|c|c|c|c|c|c|c|c|}
\hline \multirow{2}{*}{ Genótipos } & \multicolumn{5}{|c|}{$\begin{array}{c}\text { Subperíodo da emergência ao } \\
\text { espigamento }\end{array}$} & \multirow[t]{2}{*}{ Ciclo } & \multicolumn{5}{|c|}{ Altura das plantas } \\
\hline & 1996 & 1997 & 1998 & 1999 & Média & & 1996 & 1997 & 1998 & 1999 & Média \\
\hline & & & dias & 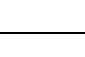 & - & & & 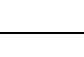 & $-\mathrm{cm}-$ & 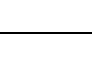 & - \\
\hline IAC 3 (Banteng) & 66 & 52 & 57 & 64 & 60 & Precoce & 95 & 96 & 103 & 97 & 98 \\
\hline STIER “S” & 70 & 52 & 62 & 71 & 64 & Médio & 106 & 97 & 102 & 98 & 101 \\
\hline IAC 2 (Tarasca) & 66 & 59 & 62 & 64 & 63 & Médio & 103 & 97 & 112 & 106 & 104 \\
\hline ARDILLA & 64 & 52 & 55 & 60 & 58 & Precoce & 96 & 90 & 106 & 97 & 97 \\
\hline IAC 1 & 66 & 55 & 57 & 64 & 61 & Médio & 110 & 96 & 113 & 103 & 106 \\
\hline ICT 8803 & 66 & 59 & 57 & 64 & 62 & Médio & 98 & 100 & 114 & 104 & 104 \\
\hline IAPAR 23 & 68 & 62 & 83 & 68 & 70 & Tardio & 103 & 97 & 106 & 104 & 102 \\
\hline CEP 15 & 62 & 52 & 55 & 59 & 57 & Precoce & 92 & 79 & 110 & 89 & 93 \\
\hline $\begin{array}{l}\text { CML"S"/PATO//KISS/DNF/3/ } \\
\text { BGL"S" / / ANTEATER }\end{array}$ & 66 & 59 & 62 & 64 & 63 & Médio & 98 & 95 & 108 & 94 & 99 \\
\hline IAC $289(t)$ & 64 & 59 & 57 & 64 & 61 & Médio & 80 & 94 & 86 & 69 & 82 \\
\hline LT 978.82/ASAD//TARASCA & 68 & 59 & 83 & 71 & 70 & Tardio & 107 & 102 & 107 & 97 & 103 \\
\hline TATU 4/ARDI 1 & 62 & 52 & 57 & 64 & 59 & Precoce & 100 & 95 & 108 & 98 & 100 \\
\hline TARASCA 87/YOGUI“S” & 68 & 59 & 57 & 64 & 62 & Médio & 106 & 86 & 112 & 104 & 102 \\
\hline $\begin{array}{l}\mathrm{BGL} / \mathrm{CIN} / / \mathrm{MUS} / 4 / \mathrm{DLF} 99 / \\
3 / \mathrm{M}^{2} \mathrm{~A} / \mathrm{F3}\end{array}$ & 66 & 55 & 55 & 64 & 60 & Precoce & 103 & 87 & 109 & 96 & 99 \\
\hline BAGAL 7 & 68 & 62 & 57 & 68 & 64 & Médio & 98 & 97 & 106 & 102 & 101 \\
\hline $\begin{array}{l}\text { CML/PATO//BGL/3/ITA BULK/ } \\
\text { 4/DRIRA/FAS204/5/YOGUI"S" }\end{array}$ & 66 & 59 & 57 & 64 & 62 & Médio & 100 & 100 & 118 & 114 & 108 \\
\hline MUS “S”*2/HARE 57 & 68 & 55 & 57 & 64 & 61 & Médio & 94 & 85 & 104 & 101 & 96 \\
\hline GRADO/ARDI 1//CIVET & 64 & 55 & 57 & 64 & 60 & Precoce & 97 & 89 & 100 & 86 & 93 \\
\hline HARE 263/CIVET "S" & 68 & 55 & 57 & 64 & 61 & Médio & 114 & 103 & 114 & 110 & 110 \\
\hline IAC $60(t)$ & 64 & 59 & 62 & 59 & 61 & Médio & 87 & 86 & 100 & 80 & 88 \\
\hline
\end{tabular}

(t) Trigo.

Os genótipos IAC 3, STIER "S", IAC 2, ICT 8803, TARASCA 87/YOGUI"S", GRADO/ARDI 1//CIVET e HARE 263/CIVET "S" apresentaram $\mathrm{b}>1$, revelando adaptabilidade específica a ambientes favoráveis e baixa estabilidade, podendo reduzir significativamente seus rendimentos em condições desfavoráveis de ambiente.

Do quadro 4 constam os resultados da avaliação da estabilidade e adaptabilidade dos genótipos em condições de várzea úmida, onde se verificou que o desvio de regressão linear foi significativo a $1 \%$ para ICT 8803, CEP 15, LT 978.82/ASAD//TARASCA e TARASCA 87/YOGUI"S" com b > 1 e CML/PATO/ /BGL/3/ITA BULK/4/DRIRA/FAS204/5/YOGUI "S" a $1 \%$ com $b<1$. O genótipo de trigo IAC 60 , com desvio de regressão significativo a $1 \%$, responsivo a ambientes desfavoráveis, apresentou a maior variação em rendimento de grãos; a diferença entre a maior e a menor média foi de $1.852 \mathrm{~kg} \cdot \mathrm{ha}^{-1}$. Essa variação deve-se ao fenômeno do chochamento dos grãos nas espigas que ocorre nessas condições ambientais, de acordo com os dados de Felicio et al. (1988) e FeLICIO et al. (1995).

Nos quadros 5 e 6 encontram-se o número de dias do subperíodo da emergência das plântulas ao espigamento pleno, o ciclo e a altura das plantas dos genótipos avaliados para os dois ambientes. O número de dias do subperíodo emergência-espigamento não foi constante; a maior duração ocorreu nas condições de várzea úmida. Segundo Magrin (1997), quando há o aumento da temperatura a taxa de desenvolvimento é acelerada, o genótipo avança mais 
rapidamente para a maturação. Esperava-se maior precocidade nas condições de cultivo de várzea úmida, pois nesse ambiente as temperaturas são mais elevadas se comparadas às condições de sequeiro com irrigação por aspersão. A umidade existente no subsolo da várzea, em decorrência dos banhos rápidos, pode ter influenciado a diferença observada.

Quanto à altura das plantas, comparando-se os dois ambientes, alguns genótipos tiveram maior crescimento nas condições de sequeiro com irrigação, não sendo, entretanto, regra geral. Os genótipos de trigo apresentaram porte mais baixo quando comparados aos genótipos de triticale e entre estes; destacaram-se pelo porte mais baixo o CEP 15 (nos dois ambientes) e o GRADO/ARDI 1//CIVET, em condições de irrigação por aspersão.
No quadro 7 encontram-se os dados da análise da estabilidade e adaptabilidade do subperíodo emergência-espigamento para cada um dos ambientes. $\mathrm{O}$ desvio de regressão linear foi significativo para os genótipos de triticale IAPAR 23 e LT 978.82/ASAD / / TARASCA nas condições de irrigação por aspersão. Em condições de várzea, o desvio de regressão não foi significativo para os genótipos de triticale STIER "S", ARDILLA, IAC 1, IAPAR 23 e TATU 4/ ARDI 1 e para os genótipos de trigo IAC 289 e IAC 60 . Os resultados revelam que a variação no número de dias do subperíodo emergência-espigamento ocorreu em maior número de genótipos nas condições de várzea (mesmo não sendo significativa), quando comparados aos irrigados por aspersão.

Quadro 6. Número de dias entre a emergência e o espigamento, ciclo e a altura das plantas dos genótipos de triticale e trigo avaliados em condição de várzea, em Mococa (Zona H), Estado de São Paulo, de 1996 a 1999

\begin{tabular}{|c|c|c|c|c|c|c|c|c|c|c|c|}
\hline \multirow{2}{*}{ Genótipos } & \multicolumn{5}{|c|}{$\begin{array}{c}\text { Subperíodo da emergência ao } \\
\text { espigamento }\end{array}$} & \multirow{2}{*}{ Ciclo } & \multicolumn{5}{|c|}{ Altura das plantas } \\
\hline & 1996 & 1997 & 1998 & 1999 & Média & & 1996 & 1997 & 1998 & 1999 & Média \\
\hline & & & - dias & 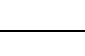 & - & & - & 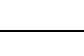 & $-\mathrm{cm}$ & 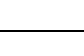 & - \\
\hline IAC 3 (Banteng) & 68 & 63 & 57 & 65 & 63 & Médio & 83 & 105 & 104 & 94 & 97 \\
\hline STIER “S” & 74 & 70 & 88 & 69 & 72 & Tardio & 88 & 115 & 111 & 84 & 100 \\
\hline IAC 2 (Tarasca) & 71 & 66 & 62 & 65 & 66 & Tardio & 82 & 126 & 131 & 95 & 107 \\
\hline ARDILLA & 76 & 58 & 55 & 60 & 62 & Médio & 85 & 112 & 100 & 92 & 97 \\
\hline IAC 1 & 74 & 63 & 57 & 65 & 65 & Médio & 71 & 125 & 106 & 109 & 103 \\
\hline ICT 8803 & 68 & 66 & 62 & 65 & 65 & Médio & 80 & 120 & 110 & 111 & 105 \\
\hline IAPAR 23 & 68 & 70 & 88 & 69 & 74 & Tardio & 72 & 115 & 120 & 97 & 101 \\
\hline CEP 15 & 68 & 58 & 55 & 59 & 60 & Precoce & 78 & 105 & 94 & 94 & 93 \\
\hline $\begin{array}{l}\text { CML“S"/PATO//KISS/DNF/ } \\
\text { 3/BGL"S" / / ANTEATER }\end{array}$ & 76 & 66 & 83 & 65 & 73 & Tardio & 83 & 110 & 107 & 98 & 100 \\
\hline IAC $289(t)$ & 74 & 66 & 88 & 65 & 73 & Tardio & 69 & 95 & 80 & 74 & 80 \\
\hline LT 978.82/ASAD//TARASCA & 74 & 70 & 83 & 69 & 74 & Tardio & 79 & 107 & 105 & 101 & 98 \\
\hline TATU 4/ARDI 1 & 76 & 63 & 57 & 59 & 64 & Médio & 80 & 106 & 107 & 99 & 98 \\
\hline TARASCA 87/YOGUI “S” & 74 & 66 & 83 & 65 & 72 & Tardio & 78 & 120 & 110 & 107 & 104 \\
\hline $\begin{array}{l}\text { BGL/CIN//MUS/4/DLF99/ } \\
3 / \mathrm{M}^{2} \mathrm{~A} / \mathrm{F3}\end{array}$ & 74 & 63 & 62 & 59 & 65 & Médio & 78 & 116 & 99 & 96 & 97 \\
\hline BAGAL 7 & 74 & 66 & 83 & 69 & 73 & Tardio & 93 & 121 & 106 & 95 & 104 \\
\hline $\begin{array}{l}\text { CML/PATO//BGL/3/ITA BULK } \\
\text { /4/DRIRA/FAS204/5/YOGUI }\end{array}$ & 74 & 66 & 83 & 65 & 72 & Tardio & 94 & 125 & 100 & 105 & 106 \\
\hline MUS“S”*2/HARE 57 & 68 & 63 & 62 & 65 & 65 & Médio & 72 & 116 & 95 & 95 & 95 \\
\hline GRADO/ARDI 1//CIVET & 71 & 66 & 62 & 65 & 66 & Médio & 83 & 112 & 104 & 102 & 101 \\
\hline HARE 263/CIVET "S" & 74 & 66 & 62 & 69 & 68 & Médio & 90 & 127 & 112 & 111 & 110 \\
\hline IAC $60(\mathrm{t})$ & 71 & 66 & 88 & 65 & 73 & Tardio & 79 & 97 & 97 & 94 & 93 \\
\hline
\end{tabular}

(t) Trigo. 
Quadro 7. Estabilidade e adaptabilidade de acordo com o modelo de EBERHART e RUSSEL(1966) e o coeficiente de determinação ( ${ }^{2}$ ) para o subperíodo da emergência ao espigamento dos genótipos de triticale e trigo avaliados em condição de irrigação por aspersão e em várzea úmida, em Mococa (Zona H), Estado de São Paulo, de 1996 a 1999

\begin{tabular}{|c|c|c|c|c|c|c|}
\hline \multirow{2}{*}{ Genótipos } & \multicolumn{3}{|c|}{ Irrigado por aspersão } & \multicolumn{3}{|c|}{ Várzea úmida } \\
\hline & $\beta_{1}{ }^{(1)}$ & $S^{2} d^{(2)}$ & $\mathrm{R}^{2}$ & $\beta_{1}{ }^{(1)}$ & $\mathrm{S} 2 \mathrm{~d}^{(2)}$ & $\mathrm{R}^{2}$ \\
\hline & — & - dias - & 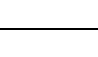 & $\longrightarrow$ & - dias - & 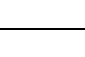 \\
\hline IAC 3 (Banteng) & $1,51 \mathrm{~ns}$ & - & 99,69 & $0,06 \mathrm{~ns}$ & - & 0,27 \\
\hline STIER “S” & $2,01 \mathrm{~ns}$ & - & 94,87 & $1,47 \mathrm{~ns}$ & $3 \mathrm{~ns}$ & 43,39 \\
\hline IAC 2 (Tarasca) & $0,69 \mathrm{~ns}$ & - & 98,07 & $0,27 \mathrm{~ns}$ & - & 8,58 \\
\hline ARDILLA & $1,22 \mathrm{~ns}$ & - & 95,99 & $1,24 \mathrm{~ns}$ & $6 \mathrm{~ns}$ & 27,23 \\
\hline IAC 1 & $1,22 \mathrm{~ns}$ & - & 95,46 & $0,46 \mathrm{~ns}$ & $4 \mathrm{~ns}$ & 6,64 \\
\hline ICT 8803 & $0,83 \mathrm{~ns}$ & - & 71,34 & $0,01 \mathrm{~ns}$ & - & 0,07 \\
\hline IAPAR 23 & $0,16 \mathrm{~ns}$ & $9^{*}$ & 0,60 & $0,94 \mathrm{~ns}$ & $8 \mathrm{~ns}$ & 15,21 \\
\hline CEP 15 & $1,02 \mathrm{~ns}$ & - & 97,78 & $0,62 \mathrm{~ns}$ & - & 19,18 \\
\hline $\begin{array}{l}\text { CML“S"/PATO/KISS/DNF/3/ } \\
\text { BGL“S"/5/ANTEATER }\end{array}$ & $0,69 \mathrm{~ns}$ & - & 98,07 & $1,93 \mathrm{~ns}$ & - & 78,57 \\
\hline IAC $289(t)$ & $0,68 \mathrm{~ns}$ & - & 66,09 & $2,05 \mathrm{~ns}$ & $4 \mathrm{~ns}$ & 57,58 \\
\hline LT 978.82/ASAD//TARASCA & $0,59 \mathrm{~ns}$ & $10^{*}$ & 6,58 & $1,18 \mathrm{~ns}$ & - & 52,84 \\
\hline TATU 4/ARDI 1 & $1,20 \mathrm{~ns}$ & - & 90,84 & $1,08 \mathrm{~ns}$ & $5 \mathrm{~ns}$ & 24,97 \\
\hline TARASCA 87/YOGUI “S” & $0,98 \mathrm{~ns}$ & - & 71,74 & $1,76 \mathrm{~ns}$ & - & 68,44 \\
\hline $\begin{array}{l}\text { BGL/CIN//MUS/4/DLF99/ } \\
3 / \mathrm{M}^{2} \mathrm{~A} / \mathrm{F} 3\end{array}$ & $1,27 \mathrm{~ns}$ & - & 86,97 & $1,20 \mathrm{~ns}$ & - & 51,87 \\
\hline BAGAL 7 & $0,88 \mathrm{~ns}$ & - & 50,59 & $1,46 \mathrm{~ns}$ & - & 59,64 \\
\hline $\begin{array}{l}\text { CML/PATO//BGL/3/ITA BULK/ } \\
\text { 4/DRIRA/FAS204/5/ YOGUI "S" }\end{array}$ & $0,83 \mathrm{~ns}$ & - & 71,90 & $1,76 \mathrm{~ns}$ & - & 68,44 \\
\hline MUS “S”*2/HARE 57 & $1,37 \mathrm{~ns}$ & - & 93,57 & $0,29 \mathrm{~ns}$ & - & 11,53 \\
\hline GRADO/ARDI 1//CIVET & $1,06 \mathrm{~ns}$ & - & 93,85 & $0,28 \mathrm{~ns}$ & - & 8,58 \\
\hline HARE 263/CIVET "S" & $1,37 \mathrm{~ns}$ & - & 93,57 & $0,24 \mathrm{~ns}$ & - & 3,51 \\
\hline IAC $60(t)$ & $0,30 \mathrm{~ns}$ & - & 27,56 & $1,79 \mathrm{~ns}$ & $7 \mathrm{~ns}$ & 43,53 \\
\hline
\end{tabular}

$\left(\begin{array}{l}1 \\ )\end{array}\right)$ Coeficiente de regressão linear; ${ }^{*} \mathrm{e}^{* *}$ : significativo a $5 \%$ e a $1 \%$ de probabilidade, respectivamente, pelo teste de Tukey, ${ }^{2}$ ) Desvio de regressão; ${ }^{*} \mathrm{e}^{* *}$ : significativo a $5 \%$ e a $1 \%$ de probabilidade, respectivamente, pelo teste $\mathrm{F}$.

(t) Trigo. -: Desvio de regressão menor que o erro experimental. ns = Não significativo.

Essa diferença pode determinar, em um genótipo, maior ou menor rendimento de grãos nas condições de várzea, em comparação ao ambiente irrigado por aspersão, pois a correlação entre as altas temperaturas e a baixa umidade relativa do ar, por ocasião do florescimento do trigo, causa o fenômeno denominado "chochamento" dos grãos na espiga e a nãoformação dos grãos, causada pela esterilidade masculina. Exclui-se, nesse caso, o chochamento causado por doenças fúngicas (CAMARGO, 1976; Felicio e LEITE, 1982).

Os resultados indicam que os genótipos de trigo apresentaram adaptação específica para condições de sequeiro com irrigação por aspersão, e o grupo de triticale, adaptação mais ampla também para várzea inundada.

\section{CONCLUSÕES}

1. A existência da alta diversidade entre os dois ambientes propiciou diferenças entre os padrões de resposta dos genótipos testados, sendo superior o rendimento de grãos no ambiente irrigado por aspersão para ambas as espécies, considerando-se as médias dos genótipos e dos anos.

2. O genótipo de triticale LT 978.82/ASAD//TARASCA apresentou alto rendimento e padrão de resposta desejável nos dois ambientes. 
3. O genótipo de trigo IAC 60 mostrou-se responsivo a ambientes desfavoráveis mas com muita variação de rendimento de grãos entre os anos.

4. O ciclo das plantas, da emergência ao espigamento, foi mais longo entre os genótipos de triticale nas condições de várzea úmida, quando comparado ao cultivo irrigado por aspersão.

\section{REFERÊNCIAS BIBLIOGRÁFICAS}

ALBERTONI, L.; BAUERMANN, N.C. Programa de triticale SADIA. In: REUNIÃO BRASILEIRA DE TRITICALE, 4., 1992. Chapecó. Anais... Chapecó: EPAGRI, 1995. 159p.

BAIER, A.C. Potencial of triticale in Southern Brazil. In: SYMPOSIUM, 2, 1990. Passo Fundo. Proceedings... México: CIMMYT/EMBRAPA-CNPT/ITA, 1991. p.9-13.

BAIER, A.C. Potencialidade do triticale no Brasil. In: REUNIÃO BRASILEIRA DE TRITICALE, 4., 1992. Chapecó, Anais... Chapecó: EPAGRI, 1995. 159p.

BAIER, A.C.; NEDEL; J.L., REIS, E.M.; WIETHOLTER, S. Triticale: cultivo e aproveitamento. 2.ed. Passo Fundo: EMBRAPA/CNPT, 1996. 72p. (EMBRAPA-CNPT, Documentos, 19)

BAKER, R.J. Genotype-environment interactions in yield of wheat. Canadian Journal of Plant Science, Otawa, v.49, p.743-751, 1969.

CAMARGO, C.E. de O. Ocorrência do chochamento em espigas de trigo no Estado de São Paulo. Bragantia, Campinas, v.35, n.10, p.107-113, 1976.

CAMPINAS, INSTITUTO AGRONÔMICO. Recomendações técnicas de trigo para 1999. 2.ed.atual. Campinas, 1999. 100p. (Boletim Técnico, 167)

CRUZ, C.D.; REGAZZI, A.J. Modelos biométricos aplicados ao melhoramento genético. Viçosa: Imprensa Universitária, 1994. 390p.

EBERHART, S.A.; RUSSELL,W.A. Stability parameters for comparing varieties. Crop Science, Madison, v.6, p.3640, 1966.
FELICIO, J.C.; CAMARGO, C.E. de O.; GALLO, P.B.; CAMARGO, M.B.P. de. Avaliação de genótipos de triticale em solos de várzea no Estado de São Paulo. Pesquisa Agropecuária Brasileira, Brasília, v.30, n.7, p.939-946, 1995.

FELICIO, J.C.; CAMARGO, C.E. de O.; LEITE, N. Avaliação de genótipos de triticale em solos de várzea no Estado de São Paulo no período de 1979 a 1984. Pesquisa Agropecuária Brasileira, Brasília, v.23, n.1, p.45-51, 1988.

FELICIO, J.C.; LEITE, N. Micronutrientes na forma de óxidos silicatados e chochamento em trigo de várzea. Pesquisa Agropecuária Brasileira, Brasília, v.17, n.10, p.1417-1421, 1982.

GOMES, F.P. Curso de estatística experimental. 4.ed. Piracicaba: Nobel, 1970. 430p.

MAGRIN, G.O. Introducción al modelo de simulación CERES-Wheat y ejemplos de aplicación en Argentina. In: KOHLI, M.M. y D. MARTINO (Eds.). 1998. Explorando altos rendimientos de trigo. La Estanzuela, Uruguay: CIMMYT-INIA, 1997. p.111-125.

RAIJ, B. van; CANTARELLA, H.; QUAGGIO, J.A.; FURLANI, A.M.C. Recomendações de adubação e calagem para o Estado de São Paulo. Campinas: Instituto Agronômico/Fundação IAC, 1996. 285p. (Boletim Técnico, 100)

SILVA, A.R. da; ANDRADE, J.M.V. de; LEITE, J.C. Trabalhos com trigo, cevada e triticale no CPAC em 1980: experimentos de micronutrientes em solo virgem de cerrado para a cultura do trigo. In: REUNIÃO DA COMISSÃO NORTE-BRASILEIRA DE PESQUISA DE TRIGO, 7., 1980, Ponta Grossa. Ata... p.59-70. (mimeografado)

SILVA, E.M.; LUCHIARI JUNIOR, A.; GUERRA. A.F.; GOMIDE, R.L. Recomendações sobre manejo de irrigação em trigo para a região dos cerrados. In: REUNIÃO DA COMISSÃO NORTE-BRASILEIRA DE PESQUISA DE TRIGO, 10., 1984. Campinas. 1984. Ata.... Brasília: EMBRAPA-CPAC, 1984.60p. 